\title{
BOUNDARY OF \\ A HOMOGENEOUS JORDAN DOMAIN
}

\author{
JUKKA SARVAS
}

A Jordan domain $D$ in the extended complex plane $\bar{C}$ is called a quasidisk if $D$ is the image of an open unit disk under a quasiconformal (abbreviated qc) mapping of $\bar{C}$ onto itself. For the basic properties of qc mappings we refer to [3]. Quasidisks can be characterized in several different ways which illustrate the multitude of the interesting properties of these domains [2].

One of the characteristic properties is homogeneity. Let $\mathscr{F}(K)$ be the family of all $K$-qc mappings $f: \bar{C} \rightarrow \bar{C}$ with $1 \leqq K<\infty$. We say that a set $E \subset \bar{C}$ is homogeneous with respect to $\mathscr{F}(K)$ if for each $z_{1}, z_{2} \in E$ there is an $f \in \mathscr{F}(K)$ with $g(E)=E$ and $f\left(z_{1}\right)=z_{2}$. Erkama showed in [1] that a domain $D$ is a quasidisk if and only if the boundary $\partial D$ of $D$ is a Jordan curve which is homogeneous with respect to $\mathscr{F}(K)$ for some $K$. This result raised the question if $\partial D$ can be replaced by $D$ in this characterization. In the present paper we answer the question affirmatively and prove the following result.

Theorem. A Jordan domain $D$ is a quasidisk if and only if $D$ is homogeneous with respect to $\mathscr{F}(K)$ for some $K, 1 \leqq K<\infty$.

Especially, this result shows that the boundary of a homogeneous Jordan domain is a quasicircle, i.e., the image of the unit circle under a qc mapping of $\bar{c}$ onto itself.

To prove the theorem we note that a quasidisk is always homogeneous with respect to $\mathscr{F}(K)$ for some $K$ because a disk is homogeneous with respect to Möbius transformations of $\bar{C}$. It remains to prove the sufficiency. By an auxiliary Möbius transformation we may suppose that $D$ lies in the finite complex plane $C$. Suppose that $D$ is homogeneous with respect to $\mathscr{F}(K)$ with $1 \leqq K<\infty$ but $D$ is not a quasidisk. Then its boundary cannot be a quasicircle and cannot have the three point property, i.e., we can find a sequence $\left(u_{i}, v_{i}, w_{i}\right), i=1,2, \ldots$, of triples of distinct points $u_{i}, v_{i}, w_{i} \in \partial D$ such that

(i) if $J_{i}$ and $J_{i}^{\prime}$ are the components of $\partial D \backslash\left\{u_{i}, v_{i}\right\}$ and $\operatorname{diam}\left(J_{i}\right) \leqq \operatorname{diam}\left(J_{i}^{\prime}\right)$, then $w_{i} \in J_{i}$ and

$$
\lim _{i \rightarrow \infty} \frac{\left|w_{i}-v_{i}\right|}{\left|u_{i}-v_{i}\right|}=\infty .
$$

In the rest of the paper we show that this situation leads to a contradiction. 
We may suppose that each open segment of line $\left(u_{i}, v_{i}\right)$ is contained either in $D$ or in int $(C \backslash D)$, the interior of $C \backslash D$. To see this, suppose that $\left|v_{i}-w_{i}\right| /\left|v_{i}-u_{i}\right|>2$. Let $z_{i} \in J_{i}^{\prime}$ with $2\left|v_{i}-z_{i}\right| \geqq\left|v_{i}-w_{i}\right|>2\left|v_{i}-u_{i}\right|=4 r_{i}$. We denote by $B(z, r)$ the open disk with center at $z \in C$ and radius $r>0$. The closure of a set $E \subset \bar{C}$ is denoted by $\bar{E}$. Next we set $\bar{B}_{i}=\bar{B}\left((1 / 2)\left(u_{i}+v_{i}\right), r_{i}\right)$. Let $I_{i}$ and $I_{i}^{\prime}$ be the components of $\partial D \backslash\left\{w_{i}, z_{i}\right\}$. Let $a_{i} \in I_{i}, b_{i} \in I_{i}^{\prime}$ be two points such that

$$
\left|a_{i}-b_{i}\right|=\operatorname{dist}\left(\bar{I}_{i} \cap \bar{B}_{i}, \bar{I}_{i}^{\prime} \cap \bar{B}_{i}\right)>0,
$$

where we have denoted the distance between two sets $A, B \subset C$ by $\operatorname{dist}(A, B)=$ inf $\left\{\left|z_{1}-z_{2}\right|: z_{1} \in A, z_{2} \in B\right\}$. Then $\left(a_{i}, b_{i}\right)$ lies entirely in $D$ or int $(C \backslash D), w_{i}$ and $z_{i}$ are in different components of $\partial D \backslash\left\{a_{i}, b_{i}\right\}$ and $\left|z_{i}-b_{i}\right| /\left|a_{i}-b_{i}\right|$ and $\left|w_{i}-b_{i}\right| /\left|a_{i}-b_{i}\right|$ tend to $\infty$ as $i$ tends to $\infty$. Finally, replace $u_{i}$ and $v_{i}$ by $a_{i}$ and $b_{i}$ (and replace $w_{i}$ by $z_{i}$ if necessary).

Note also that if $a_{i}$ and $b_{i}$ are as above and $H_{i}$ and $H_{i}^{\prime}$ are the two open half disks of $B\left((1 / 2)\left(a_{i}+b_{i}\right),(1 / 2)\left|a_{i}-b_{i}\right|\right)$ with $\left(a_{i}, b_{i}\right)$ as a common boundary, then one of them, say $H_{i}$, lies in $\bar{B}_{i}$, and, therefore, it lies in $D$ or $C \backslash \bar{D}$ because it does not meet $\bar{I}_{i} \cup \bar{I}_{i}^{\prime}=\partial D$. Recall that after the above replacement $u_{i}=a_{i}$ and $v_{i}=b_{i}$. By passing to a subsequence, if necessary, we can divide the proof into the following two cases:

Case A: Every $\left(u_{i}, v_{i}\right) \subset D$ and also the half disk $H_{i} \subset D$.

Case B: Every $\left(u_{i}, v_{i}\right) \subset \operatorname{int}(C \backslash D)$.

We first treat Case A. Fix $a \in D$. Let again $z_{i} \in J_{i}^{\prime}$ with $2\left|z_{i}-v_{i}\right| \geqq\left|w_{i}-v_{i}\right|$. Let $y_{i}^{\prime}=(1 / 2)\left(u_{i}+v_{i}\right)$ and let $y_{i}$ be the middle point of the line segment which joins $y_{i}^{\prime}$ and the middle point of the circular arc in the boundary of $H_{i}$. Let $f_{i} \in \mathscr{F}(K)$ such that $f_{i}(a)=y_{i}$ and $f_{i} D=D$. Let $L_{i}: \bar{C} \rightarrow \bar{C}$ be an affine conformal mapping such that $L_{i} H_{i}=\{z \in C:|z|<1, \operatorname{Re} z<0\}=H$ and $L_{i}\left(u_{i}\right)=-e_{2}, L_{i}\left(v_{i}\right)=e_{2}=(0,1)$, where $H$ is the open half disk $\{z \in C:|z|<1, \operatorname{Re} z<0\}$. Let $g_{i}=L_{i} \circ f_{i}, i=1,2, \ldots$ Then $b=g_{i}(a)=(-1 / 2,0), i=1,2, \ldots$. Note also that $\left|L_{i}\left(w_{i}\right),\right| L_{i}\left(z_{i}\right) \mid$ tend to infinity as $i$ tends to infinity because $\left|w_{i}-v_{i}\right| /\left|u_{i}-v_{i}\right|=\left|L_{i}\left(w_{i}\right)-e_{2}\right| / 2$ tends to infinity as $i$ tends to infinity.

The family $\left\{g_{i} \mid D: i=1,2, \ldots\right\}$ is normal because every $g_{i} \mid D$ omits points $e_{2},-e_{2}$ and $\infty$. Then we may suppose that $\lim _{i \rightarrow \infty} g_{i}=g: D \rightarrow \bar{C}$ locally uniformly in $D$. Because $H \subset g_{i} D$ and $g_{i}(a)=b \in H$ for all $i, g$ is not constant, and therefore, $g$ is a $K$-qc mapping from $D$ onto $D^{\prime} \supset H$. Because the sequence $\left\{g_{i}\right\}$ converges locally uniformly to $g$ in $D$ and $g$ is injective in $D$, then $\left\{g_{i}\right\}$ is a normal family in $\bar{C}$, and we may assume that $\left\{g_{i}\right\}$ converges to a $K$-qc mapping $h: \bar{C} \rightarrow \bar{C}$ uniformly in $\bar{C}$. Then $h \mid D=g$, and we extend $g$ to $\bar{C}$ by setting $g=h$. We may also suppose that

$$
\begin{array}{ll}
\lim _{i \rightarrow \infty} f_{i}^{-1}\left(u_{i}\right)=\tilde{u} \in \partial D, & \lim _{i \rightarrow \infty} f_{i}^{-1}\left(v_{i}\right)=\tilde{v} \in \partial D, \\
\lim _{i \rightarrow \infty} f_{i}^{-1}\left(w_{i}\right)=\tilde{w} \in \partial D, & \lim _{i \rightarrow \infty} f_{i}^{-1}\left(z_{i}\right)=\tilde{z} \in \partial D .
\end{array}
$$


Then by the construction of $g, g(\tilde{u})=-e_{2}, g(\tilde{v})=e_{2}$ and $g(\tilde{w})=\infty=g(\tilde{z})$, and therefore, $\tilde{u}, \tilde{v}$ and $\tilde{w}$ are distinct, but $\tilde{z}=\tilde{w}$.

On the other hand, let $I_{i}$ and $I_{i}^{\prime}$ be the components of $\partial D \backslash\left\{f_{i}^{-1}\left(u_{i}\right), f_{i}^{-1}\left(v_{i}\right)\right\}$ such that $f_{i}^{-1}\left(w_{i}\right) \in I_{i}$ and $f_{i}^{-1}\left(z_{i}\right) \in I_{i}^{\prime}$. Let $I$ and $I^{\prime}$ be the components of $\partial D \backslash\{\tilde{u}, \tilde{v}\}$ labelled so that $I_{i}$ tends to $I$ and $I_{i}^{\prime}$ tends to $I^{\prime}$ as $i$ tends to $\infty$. Then $\tilde{w} \in I$ (because $\tilde{w} \neq \tilde{u}, \tilde{v})$ and $\tilde{z} \in \bar{I}^{\prime}$. But $I \cap \bar{I}^{\prime}=\emptyset$ and thus $\tilde{z} \neq \tilde{w}$, which is a contradiction.

Next we treat Case B. Here every $\left(u_{i}, v_{i}\right) \subset C \backslash \bar{D}$. We may assume that $\left|w_{i}-v_{i}\right|=\sup \left\{\left|w-v_{i}\right|: w \in \bar{J}_{i}\right\}>\left|u_{i}-v_{i}\right|$ for all $i$. Then it is not difficult to see that for every $i$ there is $r>0$ such that

$$
B\left(y_{i}, r\left|w_{i}-v_{i}\right|\right) \subset D \text { with } y_{i}=w_{i}+r\left(w_{i}-v_{i}\right) .
$$

Next we observe that, by passing to a subsequence, we may assume that (1) is true for all $i$ and for a fixed $r>0$. Namely, if this is not the case, we have a decreasing sequence $r_{i}, i \geqq i_{0}$, with $\lim _{i \rightarrow \infty} r_{i}=0$ and

$$
B_{i}=B\left(y_{i}, r_{i}\left|w_{i}-v_{i}\right|\right) \subset D \text { and } \quad w_{i}^{\prime} \in\left(\partial D \backslash\left\{w_{i}\right\}\right) \cap \bar{B}_{i}
$$

for all $i \geqq i_{0}$. Here $w_{i}^{\prime} \in J_{i}^{\prime}$ because $w_{i}$ is the furthest point on $J_{i}$ from $v_{i}$. But now the points $w_{i}^{\prime}, w_{i}, v_{i}, i \geqq i_{0}$, form triples which reduce the situation to Case A because $\left(w_{i}^{\prime}, w_{i}\right) \subset D$ and if $I_{i}$ and $I_{i}^{\prime}$ are the components of $\partial D \backslash\left\{w_{i}^{\prime}, w_{i}\right\}$, then

$$
\begin{gathered}
\frac{\min \left\{\operatorname{diam}\left(I_{i}\right), \operatorname{diam}\left(I_{i}^{\prime}\right)\right\}}{\left|w_{i}^{\prime}-w_{i}\right|} \geqq \frac{\min \left\{\left|u_{i}-w_{i}\right|,\left|v_{i}-w_{i}\right|\right\}}{\left|w_{i}^{\prime}-w_{i}\right|} \\
\geqq \frac{\left|v_{i}-w_{i}\right|-\left|u_{i}-v_{i}\right|}{2 r_{i}\left|v_{i}-w_{i}\right|} \rightarrow \infty \quad \text { as } \quad i \rightarrow \infty .
\end{gathered}
$$

Therefore, we may assume that (1) is true for a fixed $r>0$ and all $i \geqq 1$.

Fix $a \in D$. Let $f_{i} \in \mathscr{F}(K)$ such that $f_{i}(a)=y_{i}$ and $f_{i} D=D, i=1,2, \ldots$, where $y_{i}$ is as in (1). Let $L_{i}$ be an affine conformal mapping such that $L_{i} B\left(y_{i}, r\left|w_{i}-v_{i}\right|\right)=$ $B(0,1)$ and $L_{i}\left(w_{i}\right)=e_{2}=(0,1)$. Then $L_{i}\left(v_{i}\right)=(1+1 / r) e_{2}$. Let $g_{i}=L_{i} \circ f_{i}, i=1,2, \ldots$ The family $\left\{g_{i} \mid D\right\}$ is a normal family because every $g_{i} \mid D$ omits points $e_{2},(1+1 / r) e_{2}$ and $\infty$. Therefore, we may assume that the sequence $\left\{g_{i}\right\}$ converges to a mapping $g: D \rightarrow C$ locally uniformly in $D$. Here $g$ cannot be constant because $B(0,1) \subset g_{i} D$ and $0=g_{i}(a)$ for all $i \geqq 1$. Therefore, $g: D \rightarrow D^{\prime}$ is $K$-qc, and we can extend it $K$-quasiconformally to $\bar{C}$ as in Case A, and we may assume that $\lim _{i \rightarrow \infty} g_{i}=g$ uniformly in $\bar{C}$.

Let $z_{i}$ be the last point where the ray $\left\{w_{i}+t\left(w_{i}-v_{i}\right): t \geqq 0\right\}$ meets $\partial D$. Then $z_{i} \in J_{i}^{\prime}$. We may suppose that

$$
\begin{array}{ll}
\lim _{i \rightarrow \infty} f_{i}^{-1}\left(u_{i}\right)=\tilde{u} \in \partial D, & \lim _{i \rightarrow \infty} f_{i}^{-1}\left(v_{i}\right)=\tilde{v} \in \partial D, \\
\lim _{i \rightarrow \infty} f_{i}^{-1}\left(w_{i}\right)=\tilde{w} \in \partial D, & \lim _{i \rightarrow \infty} f_{i}^{-1}\left(z_{i}\right)=\tilde{z} \in \partial D .
\end{array}
$$

Because $\left|g_{i}\left(f_{i}^{-1}\left(u_{i}\right)\right)-g_{i}\left(f_{i}^{-1}\left(v_{i}\right)\right)\right|=\left|L_{i}\left(u_{i}\right)-L_{i}\left(v_{i}\right)\right| \rightarrow 0$ as $i \rightarrow \infty$, we have $g(\tilde{u})=$ $g(\tilde{v})$, and thus $\tilde{u}=\tilde{v}$. Furthermore, $\quad g_{i}\left(f_{i}^{-1}\left(v_{i}\right)\right)=(1+1 / r) e_{2}, \quad g_{i}\left(f_{i}^{-1}\left(w_{i}\right)\right)=e_{2}$ 
and $g_{i}\left(f_{i}^{-1}\left(z_{i}\right)\right)=s_{i} e_{2}$ with $s_{i}<0$ for all $i \geqq 1$. Thus $g(\tilde{v}), g(\tilde{w})$ and $g(\tilde{z})$ are distinct points, and so are $\tilde{v}, \tilde{w}$ and $\tilde{z}$.

Finally, let $I_{i}$ and $I_{i}^{\prime}$ be the components of $\partial D \backslash\left\{w_{i}, z_{i}\right\}$ such that $u_{i} \in I_{i}$ and $v_{i} \in I_{i}^{\prime}$, and let $I$ and $I^{\prime}$ be the components of $\partial D \backslash\{\tilde{w}, \tilde{z}\}$ such that $f_{i}^{-1} I_{i} \rightarrow I$ and $f_{i}^{-1} I_{i}^{\prime} \rightarrow I^{\prime}$ as $i \rightarrow \infty$. Then $f_{i}^{-1}\left(u_{i}\right) \in f_{i}^{-1} I_{i}$ and $f_{i}^{-1} I_{i} \rightarrow I$ as $i \rightarrow \infty$. This implies that $\tilde{u} \in \bar{I}$. Further, $f_{i}^{-1}\left(v_{i}\right) \in f_{i}^{-1} I_{i}^{\prime}$ and $f_{i}^{-1} I_{i}^{\prime} \rightarrow I^{\prime}$ as $i \rightarrow \infty$. This implies that $\tilde{v} \in \bar{I}^{\prime}$. Because $\tilde{v} \neq \tilde{w}, \tilde{z}$, we have $\tilde{v} \in I^{\prime}$. Since $\bar{I} \cap I^{\prime}=\emptyset$, we have $\tilde{u} \neq \tilde{v}$, which is a contradiction. The theorem is proved.

\section{References}

[1] Erkama, T.: Quasiconformally homogeneous curves. - Michigan Math. J. 24, 1977, 157-159.

[2] Gehring, F. W.: Characteristic properties of quasidisks. - Les Presses de l'Université de Montréal, Montréal, 1982.

[3] Lehto, O., and K. I. Virtanen: Quasiconformal mappings in the plane. - Springer-Verlag, Berlin-Heidelberg-New York, 1973.

University of Helsinki

Department of Mathematics

SF-00100 Helsinki

Finland

Received 11 September 1984 